Article abstract-The presence of extrapyramidal signs or psychosis may indicate greater disability in patients with probable Alzheimer's disease. We evaluated the ability of these signs, noted at a patient's first visit, to predict one of two specific clinical endpoints: (1) a preselected score on the modified Mini-Mental State examination (cognitive endpoint), and (2) a preselected score on the Blessed Dementia Rating Scale (functional endpoint). Sixty-five patients were followed either until they reached the endpoints or to the end of the study period. Survivorship curves were drawn to predict the distribution of time to onset of an endpoint in patients with and without the clinical signs. Time to reach the cognitive endpoint was shorter for patients with extrapyramidal signs or psychosis compared with those without these signs and symptoms. These clinical signs did not predict the functional endpoint. We conclude that extrapyramidal signs and psychosis may be useful predictors of intellectual decline in Alzheimer's disease.

\title{
Predictors of disease course in patients with probable Alzheimer's disease
}

\author{
Y. Stern, PhD; R. Mayeux, MD; M. Sano, PhD; W.A. Hauser, MD; and T. Bush, PhD
}

It is difficult to predict when an individual patient with Alzheimer's disease will reach a critical stage, such as inability to perform simple daily activities, because the rate of progression varies. The clinician should be able to provide the patient and family with a reliable prediction of the course of the disease but this is not now possible.

We and others found that certain clinical signs in Alzheimer's disease may indicate more severe disease. ${ }^{1-4}$ Patients with myoclonus, extrapyramidal signs, or psychosis are more functionally and intellectually impaired.1-3

We evaluated the ability of these clinical signs, when noted at a patient's first evaluation, to predict when the patient would reach a specific clinical endpoint. We chose two endpoints: (1) impairment of intellect, or (2) functional impairment in ability to perform activities of daily living.

Methods. Subjects. Sixty-five patients with probable Alzheimer's disease (pAD), followed for up to 7.1 years (mean 2.8 \pm 1.6$)$, had mean age at onset of 63.8 $( \pm 9.5)$, and mean years of education of $12.9( \pm 3.7)$. There were 29 men and 36 women. Many of these patients were included in a previous study,,$^{1}$ but they have now been followed for a longer time, and 15 other patients have been added. All met DSM-III criteria for primary degenerative dementia ${ }^{5}$ and NINCDS-ADRDA criteria for "probable" Alzheimer's disease. ${ }^{6}$ All patients were followed for at least 6 months. We excluded patients with a history of affective disorder within 1 year of onset of intellectual decline; Parkinson's disease; history or evidence of stroke or Hachinski Ischemic score of 5 or more ${ }^{7}$; history or clinical evidence of cancer, or of car- diac, hepatic, pulmonary, or renal disease; history of anoxia or metabolic-toxic encephalopathy; or seizures preceding onset of dementia.

Procedure. All patients were evaluated at least twice, with a minimum interval of 6 months between evaluations. Each evaluation consisted of standardized neurologic, neuropsychological, and psychiatric examinations. Historical information was collected at the first evaluation.

Neurologic assessment. The presence of extrapyramidal symptoms, including tremor, rigidity, bradykinesia, gait, postural change and facial appearánce, was determined and their severity rated on a 0 to 4 scale $(0=$ none, $4=$ greatest severity) using an abbreviated version of the Columbia University Parkinson's Disease Evaluation. ${ }^{8}$ The reliability of this scale has been established. ${ }^{9}$ Myoclonus and other dyskinesias were noted and rated on a similar scale. All medications were recorded. If the patient took any medication that might have caused extrapyramidal signs, these were considered to be drug-induced.

Capacity for independence in activities of daily life was rated on part 1 of the Blessed Dementia Rating Scale (BDRS). ${ }^{10}$ Scores on this scale increase as functional ability decreases.

At the first visit, the patients and reliable informants were interviewed to determine the date at which the patient first met criteria for the diagnosis of pAD. The reliability of our onset assessments was confirmed using standardized interview techniques. ${ }^{11}$

Neuropsychological assessment. General intellectual function was measured by a modified version of the Mini-Mental State Examination (mMMS) ${ }^{12,13}$ Maximum score is 57 ; a decrease in score is indicative of a

From the Departments of Neurology (Drs. Stern, Mayeux, Sano, and Hauser), Psychiatry (Drs. Stern and Mayeux), and Epidemiology (Drs. Hauser and Bush), and the Sergievsky Center (Dr. Hauser), Columbia University College of Physicians and Surgeons, New York, NY.

Supported by the Charles S. Robertson Memorial Gift for Alzheimer's Disease Research, the Marble Fund, and federal grants AG02802 and AG05433. Patients were seen in the outpatient clinics of the General Clinical Research Center and data were stored and analyzed on a CLINFO system (both RR00645).

Received August 5, 1986. Accepted for publication in final form January 20, 1987.

Address correspondence and reprint requests to Dr. Stern, Neurological Institute, 710 West 168th Street, New York, NY 10032. 
Table. Mean mMMS and BDRS scores of patients with and without clinical signs at their first visit

\begin{tabular}{|c|c|c|c|c|}
\hline \multirow[b]{2}{*}{ Group } & \multicolumn{2}{|c|}{ mMMS } & \multicolumn{2}{|c|}{ BDRS } \\
\hline & Mean & SD & Mean & SD \\
\hline No extrapyramidal signs & 31.9 & 14.7 & 8.1 & 4.4 \\
\hline Extrapyramidal signs & 20.8 & $13.9^{*}$ & 10.8 & $4.4^{*}$ \\
\hline No psychosis & 29.9 & 15.9 & 8.7 & 4.7 \\
\hline Psychosis & 19.7 & $11.5^{*}$ & 10.6 & 3.7 \\
\hline No myoclonus & 28.1 & 15.2 & 9.1 & 4.6 \\
\hline Myoclonus & 13.3 & $18.2^{*}$ & 10.6 & 3.7 \\
\hline
\end{tabular}

decline in intellectual function. The mMMS correlates well with the original MMS and with more detailed neuropsychological examinations. ${ }^{14}$

Psychiatric assessment. All subjects and available family members or informants were interviewed in a semi-structured fashion to assess the presence of psychiatric symptoms, using the DSM-III as a guideline. The presence of persistent hallucinations, illusions, or delusions was sufficient to rate a patient as having psychosis.

Determination of endpoints. DSM-III criteria for primary degenerative dementia require both cognitive change, including primary and other intellectual functions, and functional change, defined as the inability to perform social or occupational function. Previously, we found a correlation between the mMMS and the BDRS $(\mathrm{r}=-0.5, p<0.05) .^{1}$ This suggests that although the spheres of behavior that these two tests measure are related, there are aspects of behavior that each describes uniquely and that do not co-vary. For this reason we chose to include both cognitive and functional endpoints.

The point at which the patient reached an mMMS score of less than 20 was defined as the cognitive endpoint. This score indicates poor performance on assessments of memory, orientation, language, and calculation. Reaching a BDRS score of greater than 15 was defined as the functional endpoint. A patient reaching this score generally has functional deterioration in the three separate portions of the scale: habits, personal care, and personality. These endpoints were chosen from these continuous scales because in our experience they correspond to what we consider a "moderate" level of dementia.

Analysis. As an initial estimate of the effectiveness of each predictor, chi-square analyses were used to compare the frequency of reaching an endpoint in patients with and without a specific sign at their first visit. However, this analysis does not reflect the length of time patients were followed, and cannot yield information about the distribution of time to reach an endpoint. To evaluate these issues, the Kaplan-Meier product limit technique ${ }^{15}$ was used. This maximum-likelihood nonparametric estimation procedure generates life tables that describe the probability of patients reaching an endpoint as a function of duration of illness. It takes into account the fact that some patients do not reach the endpoints during the study period.

For the purposes of this analysis, patients were followed either (1) until they reached each endpoint, or (2) until their last visit. Those patients not reaching an endpoint by their last visit were withdrawn at the time of their last visit. No patient died before reaching an endpoint. Some patients had reached one of the study endpoints before their first visit. They were excluded from the life table analyses since it is not clear whether the clinical signs were present prior to reaching the endpoint, and therefore these patients are not informative for the evaluation of predictive value.

"Survival" curves were drawn from life table data to represent the probability of reaching an endpoint as duration of illness progresses. For comparison, curves for each endpoint were plotted simultaneously for patients with and without each clinical symptom.

Results. Distribution of clinical signs at first visit. At the first visit, $24(37 \%)$ of the patients had extrapyramidal signs. Of these, four were classified as "druginduced." In none of these patients was the presence of these signs suggestive of Parkinson's disease. Psychosis, consisting primarily of delusions, was present in 17 (27\%) of the patients. Myoclonus was noted in five (7.8\%). Some patients developed extrapyramidal signs, psychosis, or myoclonus after their first visit. They were classified as being symptom-free at the first visit. Since the number of patients with myoclonus at the first visit was too small for meaningful application of the epidemiologic techniques employed in this study, the predictive utility of this clinical sign was not investigated. However, three patients from this group have died and Alzheimer's disease was confirmed and reported. ${ }^{16}$

Duration of illness was comparable in patients with and without extrapyramidal signs, psychosis, or myoclonus but, as reported previously, mMMS scores were poorer in patients with these clinical signs and BDRS was poorer in the patients with extrapyramidal signs (table).

Distribution of patients reaching endpoints. Twenty patients reached the cognitive endpoint and 6 the functional endpoint by the first visit. The distribution of patients who had reached endpoints by their first visit did not differ in patients with and without extrapyramidal signs or with and without psychosis.

Eighteen of the 20 patients with extrapyramidal signs at first visit reached the cognitive endpoint, whereas only 22 of 41 patients without these signs reached this point. Similarly, 16 of the 20 patients with these signs reached the functional endpoint as compared with 22 of the 41 without them. Chi-square analyses suggested that patients who reached the endpoints were more likely to have had extrapyramidal signs at entry (chi-square $=7.91, p<0.005$ and chi-square $=$ $3.97, p<0.05$, respectively). In patients with psychosis, 14 of 20 reached the cognitive endpoint compared with 27 of 46 without psychosis at first visit (chi-square $=$ $3.06, p<0.09$ ). Thirteen of 17 patients with psychosis reached the functional endpoint compared with 28 of 46 


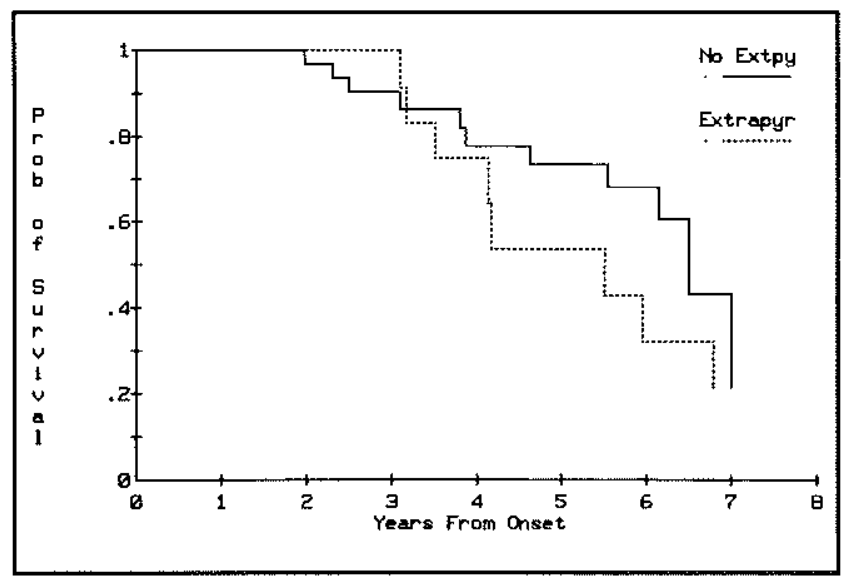

Figure 1. Probability of patients with and without extrapyramidal signs at their first visit surviving over time without reaching the endpoint of $m M M S<20$. Time is expressed in duration of illness from estimated date of onset.

patients without psychosis (chi-square $=1.33, \mathrm{~ns})$.

Life table analyses. Extrapyramidal signs. The survivorship curves in figure 1 represent the probability of reaching an mMMS score of less than 20 as a function of duration of illness. Compared with other patients, those with extrapyramidal signs at first visit were likely to reach this endpoint sooner. Patients with drug-induced extrapyramidal signs were excluded from these analyses, but similar results were obtained when these patients were included in the extrapyramidal group.

Unlike the results seen with the mMMS endpoint, there were no differences between the extrapyramidal and non-extrapyramidal groups for reaching the endpoint of BDRS > 15; the survivorship curves virtually overlap (figure 2). The median time to this endpoint in patients with and without extrapyramidal signs was 5.5 and 5.8 years, respectively.

Psychosis. Similar analyses were used to compare the duration of illness at which endpoints were reached in patients with and without psychosis. Figures 3 and 4 demonstrate that, compared with patients without psychosis, psychotic patients reached the cognitive endpoint but not the functional endpoint earlier in the course of the illness.

Additional life table analyses. Four of the patients with psychosis were receiving antipsychotics for their symptoms; four patients without psychosis were also receiving these medications for other reasons. These medications might influence mMMS or BDRS scores, but life tables were similar if patients taking these medications were excluded from the analyses.

In order to evaluate the possible contribution of myoclonus to the present findings, life tables were recalculated excluding all patients with myoclonus. Results were unchanged.

Similarly, we evaluated the independent predictive value of extrapyramidal signs and psychosis. Life tables were recalculated to compare (1) patients with and without extrapyramidal signs, with no patients in either group having psychosis, and (2) patients with and

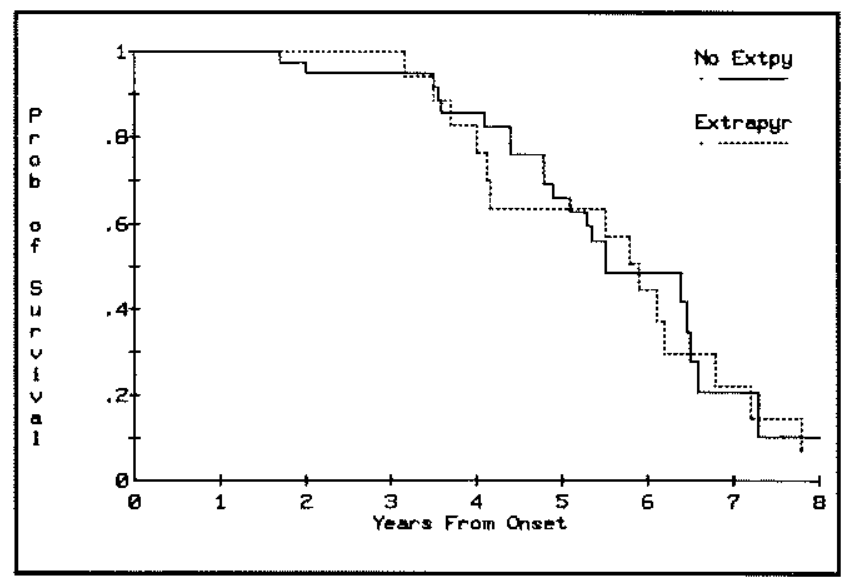

Figure 2. Probability of patients with and without extrapyramidal signs at their first visit surviving over time without reaching the endpoint of $B D R S>15$. Time is expressed in duration of illness from estimated date of onset.

without psychosis, with no patients manifesting extrapyramidal signs. Results were unchanged.

Additional analyses were done to evaluate whether patients with extrapyramidal signs or psychosis reached the endpoints more quickly because they had poorer scores on the mMMS and BDRS at the first visit. Patients were stratified into groups above and below the mean mMMS or BDRS scores at first visit. Survivorship curves were then redrawn for these stratified groups. The results were again unchanged, indicating that poor initial performance did not influence the life table results.

Discussion. Our data suggest that the presence of extrapyramidal signs or psychosis early in the disease may predict more rapid intellectual decline.

Life-table methodology is a powerful way to evaluate this type of data because it includes patients in the analysis for only the time in which they were studied. The survivorship curves are descriptive but suggest the robust predictive power of the clinical signs, particularly for the cognitive endpoint. These curves suggest that the time for reaching the cognitive endpoint is earlier in patients with extrapyramidal signs or psychosis.

While the survivorship curves suggest that patients with extrapyramidal signs or psychotic symptoms reach our endpoints more quickly, the curves do not differ significantly. Statistical power was limited by the relatively small number of patients. Other aspects of the data collection were also not optimal. Duration of illness at first visit and the interval between examinations varied between patients. In addition, time from onset of symptoms to first visit varied, and endpoints had sometimes been reached before the first visit. The data are therefore best analyzed descriptively and used to generate testable hypotheses.

The predictors were more useful for the cognitive than the functional endpoint. Both endpoints were designed to capture non-overlapping behavioral domains, 


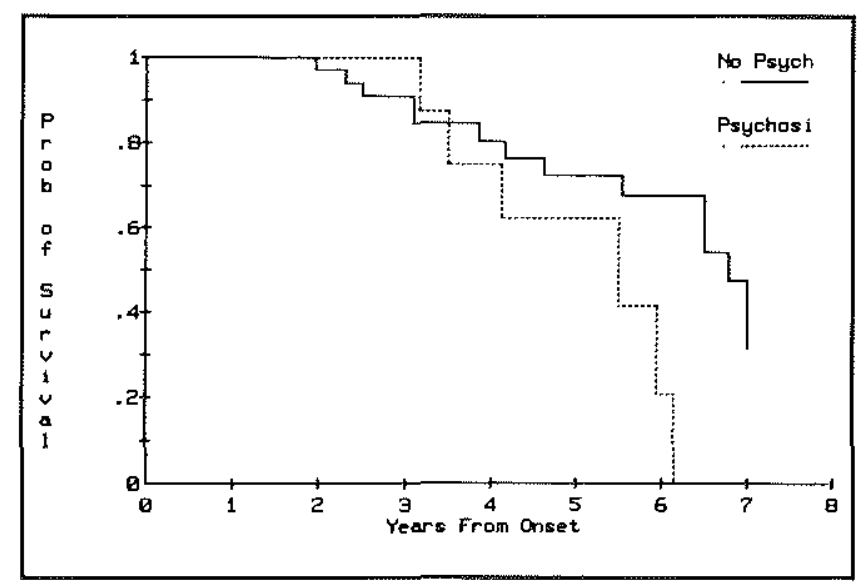

Figure 3. Probability of patients with and without symptoms of psychosis at their first visit surviving over time without reaching the endpoint of $m M M S<20$. Time is expressed in duration of illness from estimated date of onset.

and the predictors could simply have been more useful for one of them. However, it is also possible that the cutoff score we chose was not meaningful or optimally defined. We have shown that there is a relatively steady increase in BDRS scores over time, ${ }^{1}$ so this explanation seems unlikely; all patients will eventually reach any particular level of functional disability. Still, our cutoff point might reflect a degree of disability that is too extreme to be useful in delineating the utility of potential predictors. It is also possible that the BDRS is not sensitive or specific enough to capture the range of functional changes necessary to demonstrate differences between disease subgroups.

Endpoints are difficult to define since they are often dependent on multiple factors. For example, admission to a nursing home might appear to be a suitable endpoint for clinical prediction, but the severity of a patient's dementia is only one factor used to determine whether a patient is admitted into such a facility. Endpoints must be carefully defined; they must be distinct from diagnostic criteria and from the predictors. ${ }^{17}$

Recently, postmortem studies of patients who had had pAD indicated that coincident neuropathologic changes of Alzheimer's disease and Parkinson's disease, including degeneration in the substantia nigra and Lewy bodies, may exist. ${ }^{18}$ While none of the patients in that study or in two previous ones ${ }^{1,4}$ had Parkinson's disease, it is likely that the noted extrapyramidal signs are symptomatic of a more diffuse degenerative process.

Psychosis at the first visit is also associated with a greater probability of reaching the cognitive endpoint earlier. We reported these symptoms to be more common in patients with extrapyramidal signs or myoclonus, ${ }^{1}$ so it is not clear to what extent they constitute separate clinical predictors. However, psychosis and extrapyramidal signs were predictive when patients who had both clinical signs were excluded from analysis, which suggests that each clinical sign has unique predictive value.

Although there were too few patients with myo-

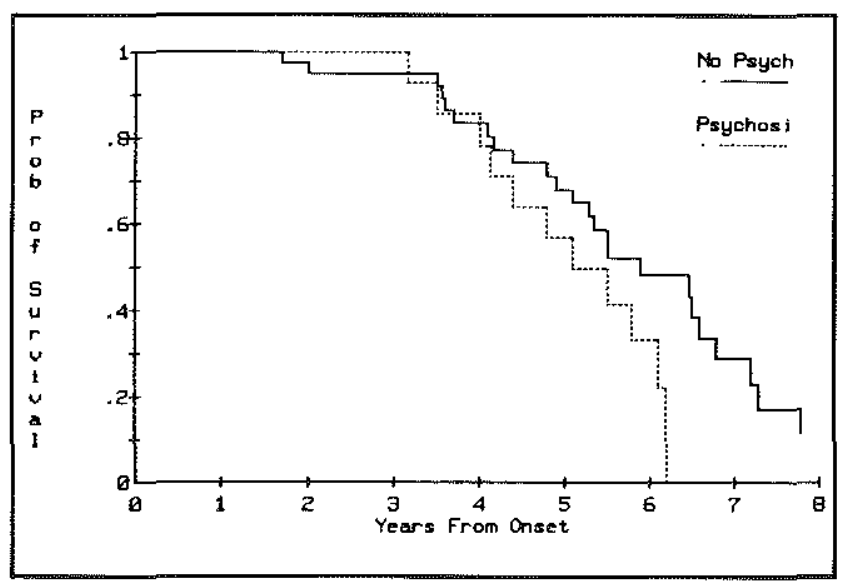

Figure 4. Probability of patients with and without symptoms of psychosis at their first visit surviving over time without reaching the endpoint of $B D R S>15$. Time is expressed in duration of illness from estimated date of onset.

clonus at the first visit to include that symptom in these analyses, this symptom is associated with a more aggressive disease course. ${ }^{1}$ An association has been $o b-$ served between autosomal dominant Alzheimer's disease with myoclonus and "spongy change" in the neocortex. ${ }^{16}$ These patients also had a greater number of neurofibrillary tangles, suggesting that the presence of myoclonus may indicate an aggressive form of Alzheimer's disease. Similar biochemical data regarding reduced choline acetyltransferase activity in this subgroup supports this view. ${ }^{19}$

These observations need confirmation and verification, but they have practical and biologic importance. Our data suggest that, at an individual patient's first assessment, a clinician may be able to predict how rapidly a certain level of intellectual dysfunction will be reached. If confirmed, this would have a significant part in the long-term care of the patient and the planning of the patient and family. Our data also suggest that variability of biologic changes in Alzheimer's disease influence its clinical expression.

\section{Acknowledgments}

We thank the staff of the Clinical Research Center Outpatient Clinic for their assistance in following the patients, and Donald McMahon for his assistance with data management.

\section{References}

1. Mayeux R, Stern Y, Spanton S. Heterogeneity in dementia of the Alzheimer type: evidence of subgroups. Neurology $1985 ; 35: 453-461$.

2. Mayeux R, Stern Y, Sano M. Psychosis in patients with dementia of the Alzheimer type. Abstract. Ann Neurol 1985;18:144.

3. Stern Y, Mayeux R. Stability and prognostic value of clinical heterogeneity in dementia of the Alzheimer type. Abstract. Neurology 1985;35(suppl 1):163.

4. Chui HC, Teng EL, Henderson VW, Moy AC. Clinical subtypes of dementia of the Alzheimer type. Neurology 1985;35:1544-1550. 
5. APA. Diagnostic and statistical manual of mental disorders, 3rd ed. The American Psychiatric Association, 1980.

6. McKhann G, Drachman D, Folstein M, et al. Clinical diagnosis of Alzheimer's disease: Report of the NINCDS-ADRDA work group under the auspices of the Department of Health and Human Services Task Force on Alzheimer's disease. Neurology 1984;34:939-944.

7. Hachinski VC, Iliff LD, Zhilka E, et al. Cerebral blood flow in dementia. Arch Neurol 1975;32:632-637.

8. Lesser RP, Fahn S, Snider SR, Cote LJ, Isgreen WP, Barrett RE Analysis of the clinical problems in parkinsonism and the complications of long-term levodopa therapy. Neurology 1979;29:1253-1260

9. Montgomery GK, Reynolds NC, Warren RM. Qualitative assessment of Parkinson's disease: study of reliability and data reduction with an abbreviated Columbia scale. Clin Neuropharmacol 1985;8:83-92.

10. Blessed G, Tomlinson BE, Roth $\mathrm{M}$. The association between quantitative measures of dementia and of senile changes in the cerebral grey matter of elderly subjects. Br J Psychol 1968;225:797-811.

11. Sano M, Stern Y, Mayeux R, Hartman S, Devanand DP. A standardized technique for establishing the onset symptoms of proba- ble Alzheimer's disease. Abstract. J Exp Clin Neuropsychol 1987;9:65-66.

12. Folstein MF, Folstein SE, McHugh PR. "Mini-mental state," practical method for grading the cognitive state of patients for the clinician. J Psychiatr Res 1975;12:189-198.

13. Mayeux R, Stern Y, Rosen J, Leventhal J. Depression, intellectual impairment and Parkinson's disease. Neurology 1981;31:645-650.

14. Stern Y, Sano M, Paulson J, Mayeux R. Modified mini-mental state examination: validity and reliability. Abstract. Neurology 1987;37(suppl 1):179.

15. Kaplan EL, Meier P. Nonparametric estimation from incomplete observations. Amer Stat Assn J 1958;53:457-481.

16. Mayeux R, Kupsky W, Duffy P. Familial Alzheimer's disease with "spongy change." Abstract. Neurology 1986;36(suppl 1):272.

17. Wasson JH, Sox HC, Neff RK, Goldman L. Clinical prediction rules: applications and methodological standards. N Engl J Med 1985;313:793-799.

18. Ditter SM, Mirra SS. Parkinson's disease features in Alzheimer's disease: a neuropathologic and clinical study, Abstract. Neurology 1986;36(suppl 1):225.

19. Bird TD, Stranahan S, Sumi M, Raskind M. Alzheimer's disease and choline acetyltransferase activity in brain tissue from clinical and pathological subgroups. Ann Neurol 1983;14:184-193. 


